\title{
Insights through Modal Minimal Models for Analysis of Linear and Nonlinear Dynamic Problems
}

\author{
Andreas Hohl $\mathbb{D}^{1},{ }^{1}$ Vincent Kulke $\mathbb{D},{ }^{2}$ and Georg-Peter Ostermeyer $\mathbb{1 D}^{2}$ \\ ${ }^{1}$ Baker Hughes, Celle, Germany \\ ${ }^{2} \mathrm{TU}$ Braunschweig, Institute of Dynamics and Vibrations, Braunschweig, Germany \\ Correspondence should be addressed to Georg-Peter Ostermeyer; gp.ostermeyer@tu-bs.de
}

Received 27 October 2021; Accepted 22 December 2021; Published 24 January 2022

Academic Editor: Stefano Marchesiello

Copyright (C) 2022 Andreas Hohl et al. This is an open access article distributed under the Creative Commons Attribution License, which permits unrestricted use, distribution, and reproduction in any medium, provided the original work is properly cited.

Dynamic analysis of three-dimensional structures is common practice in industry to optimize products and in research to gain insights on the influence of parameters. The complexity differs based on the linearity or nonlinearity of the underlying problem, the type of excitation, e.g., forced or self-excitation, and the number of degrees of freedom that need to be examined. Reduced order models and optimized numerical methods are used to optimize the time and computational power needed to gain a certain insight. This article focusses on a specific class of problems where the modes of the structure do not or do not significantly change through the (damping) device or force that is added to the structure. Herein, lumped mass models are commonly used for analysis of the dynamic response of the system. In the article, it is highlighted that lumped mass models can give quantitative insight but modally reduced models allow a direct optimization of the problems with respect to the physical degree of freedom that, for example, is subject to self-excitation or dampened. The benefit of modal minimal models and its limitations are shown and discussed for different linear and nonlinear dynamic problems.

\section{Introduction}

A dynamic analysis of complex three-dimensional structures is commonly done using numerical models with numerous degrees of freedom, whereas these models are efficient for a single linear analysis, e.g., to analyze the mechanical integrity of a structure, and they are inefficient for solving nonlinear problems or optimization problems, e.g., with contacting interfaces or the problem of the optimal placement of a nonlinear vibration mitigation device. Methods like the Harmonic Balance Method (HBM) [1], the Alternating Frequency-Time [2] (AFT) method, or conventional averaging methods [3] have been developed to determine the nonlinear response of the system, e.g., to periodical excitation. Herein, Reduced Order Models (ROMs) like the Component Mode Synthesis [4] are commonly used to represent the dynamic behavior of the structure for the specific problem and underlying parameters. These methods are especially useful if the nonlinear forces strongly influence the modes of the underlying structure or the nonlinearity cannot be approximated in a narrow frequency range. In this case, constraint modes $[4,5]$ can be used or a superposition of the modal ansatz functions/vectors that need to be able to picture the response of the nonlinear system.

The work done here is focusing on the special case that the modes of the structure are not significantly changed through the linear or nonlinear forces that are acting on the structure. In this case, lumped mass models are often used that represent a single degree of freedom (one mode) of the structure [6-10]. In contrast, this paper highlights the benefits of using modally reduced models [11-16] with one degree of freedom for this class of problems. For this purpose, different examples are shown that are usually optimized with lumped mass models or with complex threedimensional numerical models. The examples include the efficient identification of the critical modes in a self-excited system and optimization of the placement of frictional and viscous damping devices and tuned-mass dampers. The examples are discussed and are benchmarked with the lumped mass models, and the suitability and limitations are 
discussed. The focus is on problems that can be solved analytically or semianalytically, and direct insight of parameter influences can be provided.

\section{Comparing Modally Reduced and Lumped Mass Models}

A mechanical system with several degrees of freedom is typically described using complex structural models based on finite elements or partial differential equations. The goal is to find minimal modal models that quantitatively deliver the same results and can be based on the complex models. These can be derived at the cost of a numerical or experimental modal analysis but can deliver analytical equations to predict loads or to optimize damper design and strategies in linear or nonlinear systems.

The approach using a modal single degree of freedom (SDOF) oscillator (modal DOF $q$ ) and a physical single-mass oscillator (physical DOF $x_{j}$ ) is analyzed, and the results are discussed. The equation of motion of a lumped mass model is

$$
m_{j} \ddot{x}_{j}(t)+d_{j} \dot{x}_{j}(t)+c_{j} x_{j}(t)=M_{j}(t),
$$

or mass normalized as

$$
\ddot{x}_{j}(t)+2 D_{i} \omega_{0, i} \dot{x}_{j}(t)+\omega_{0, i}^{2} x_{j}(t)=\frac{1}{m_{j}} M_{j}(t) .
$$

The equation can be used to describe one mode $i$ with a modal damping value $D_{i}$ and an angular natural frequency $\omega_{0, i} . M_{j}(t)$ describes a force acting on the structure at a predefined point $j$. The ratio of the stiffness $c_{j}$ and the mass $m_{j}$ and the viscous damping $d_{j}$ need to be adjusted to account for a point of excitation $j$ with respect to the mode shape of the structure and to adjust for the modal damping value and the natural frequency. The model is only valid for the point where it is observed which has to be the same point or node $j$ where it is excited.

The modal single degree of freedom model with the modal DOF $q_{i}$ of any mode $i$ of the structure is determined directly from its mass normalized modal parameters:

$$
\ddot{q}_{i}(t)+2 D_{i} \omega_{0, i} \dot{q}_{i}(t)+\omega_{0, i}^{2} q_{i}(t)=\sum_{j=1}^{n} \varphi_{i, j} M_{j}(t),
$$

Here, $M_{j}$ is the physical force that is acting at a position $j$. The modal force at the right side of the equation is calculated by summation of the multiplication of the mass normalized mode shape amplitude $\varphi_{i, j}$ with the physical forces $M_{j}$ for each position $j$, respectively. Complex excitation can be applied at multiple positions with different phase information.

Although both minimal models are leading to similar mathematical solutions, the modal approach offers certain advantages and more direct insights. Qualitative and quantitative insights are directly gained through the modal properties that are present in the analytical equations.

In case of the modal model, forces can be applied at every node and physical amplitudes can be calculated at every point of interest $k$ by $x_{k}=\varphi_{i, k} q_{i}$ (assuming one contributing mode). The modal parameters are directly used and are not converted into substitute parameters, which is especially beneficial for investigating parameter influences such as frequency or mode shape changes as well as for parametric or statistical analysis. Furthermore, in the modally reduced model, the positions of the acting forces are directly considered in the differential equation via $\sum_{j=1}^{n} \varphi_{i, j} M_{j}(t)$ and multiple forces at multiple positions can be acting on the structure.

2.1. Critical Modes in Self-Excited Systems. The first example that highlights the benefits of a modal model is the identification of self-excited modes [13]. There are different mechanisms for self-excitation of a system described in the literature. In the case where an elastic structure interacts with an interface, a common model for self-excitation is of a velocity-dependent contact force characteristic. Figure 1. Qualitatively a negative/positive slope of the nonlinear contact force characteristic corresponds to an energy input/ energy dissipation for small monoharmonic vibrations with respect to a mean relative velocity $v_{0,1}$ or $v_{0,2}$. The absolute value of the slope scales the net energy in one period of vibration linearly. The question answered here is which mode $i$ is unstable or likely to be excited.

A modal model for each mode represented by the number $i$ is used that directly shows the influence of the point of excitation (the degree of freedom $j$ ) that is represented by the amplitude $\varphi_{i, j}$ of the mode shape. The contact force characteristic is linearized with respect to a mean relative velocity $v_{0}$.

This leads to the modal equation of motion:

$$
\ddot{q}_{i}(t)+2 D_{i} \omega_{0, i} \dot{q}_{i}(t)+\omega_{0, i}^{2} q_{i}(t)=\sum_{j=1}^{n} \varphi_{i, j}^{2} S\left(v_{0}\right) \dot{q}_{i}(t) .
$$

Here, $s$ denotes the slope of the contact force characteristic with respect to the mean relative velocity between the two interfaces $v_{0}$. Assuming only one point of excitation $j$ and one contributing mode $i$ of the structure, all velocitydependent parameters can be summarized resulting in an equivalent damping for the mode:

$$
D_{\mathrm{eq}, i}=D_{i}-\frac{\varphi_{i, j}^{2}}{2 \omega_{0, i}} S\left(v_{0}\right) .
$$

The sign of the equivalent damping is an indicator for the fix-point stability for the given parameters. The system is marginally stable if $D_{\text {eq, } i}=0$ leading to two equivalent equations as follows:

$$
\begin{gathered}
S_{\mathrm{c}, i}=-\frac{2 D_{i} \omega_{0, i}}{\varphi_{i, j}^{2}}, \\
D_{\mathrm{c}, i}=\frac{\varphi_{i, j}^{2}}{2 \omega_{0, i}} S\left(v_{0}\right) .
\end{gathered}
$$

That can be used to rank the stability of the modes with respect to the underlying self-excitation mechanism. $S_{c, i}$ is 


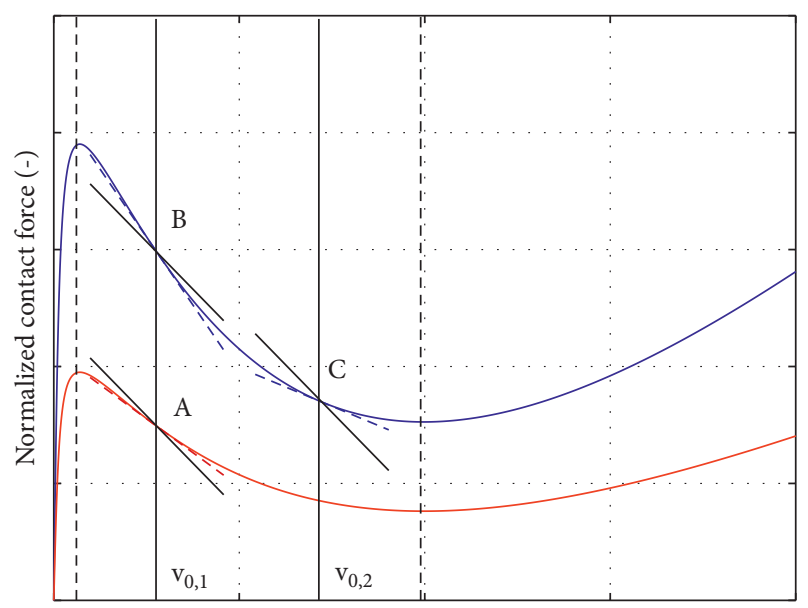

Normalized relative velocity (-)

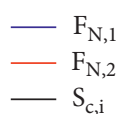

Figure 1: Example of a nonlinear contact force characteristic. The slope of the characteristic is assumed to be linearly scaled by the normal force $F_{\mathrm{N}}\left(F_{\mathrm{N}, 1}=2 \cdot F_{\mathrm{N}, 2}\right)$. Points $\mathrm{A}$ and $\mathrm{C}$ are stable/not self-excited (the slope needed $S_{c, i}$ is steeper than the actual slope of the curve. Point B is unstable/self-excited (the slope needed $S_{c, i}$ is less steep than the actual slope of the curve).

the (negative) slope of the velocity weakening contact force characteristic that needs to be exceeded (the absolute value) to get self-excitation of a mode with the modal properties $D_{i}$, $\omega_{0, i}$, and $\varphi_{i, j}^{2}$. The modal description accentuates the contribution of the amplitude $\varphi_{i, j}^{2}$ of the mode shape at the contacting interface which is not visible in case of a lumped mass model.

The concept is visualized in Figure 1. An elastic structure is regarded that can be described by a specific number of modes. The degree of freedom $j$ of the elastic structure is tangentially in contact with a rigid counterpart. The contacting interfaces at $j$ have a relative velocity $v_{0}$ and a constant normal force $F_{\mathrm{N}}$ orthogonal to $j$. The tangential force characteristic is assumed to be nonlinearly dependent on the relative velocity $v_{0}$ between the node $j$ and linearly dependent on the normal force in the contact interface (Figure 1). As an example, the stability of a specific mode $i$ is analyzed with reference to three points $\mathrm{A}, \mathrm{B}$, and $\mathrm{C}$ in Figure 1. In these points, the critical slope of the mode $S_{\mathrm{c}, i}$ is related to the slope of the contact force characteristic. In point $\mathrm{A}$, the relative velocity and the normal force are comparably low. The slope of the contact force characteristic is less negative compared with the mode specific $S_{c, i}$ value which is the slope of the black line through A. Therefore, following the logics of (5) and (6), not enough energy input is provided to overcome the energy dissipation through the modal damping ( $1 \%$ in the example). Therefore, point $\mathrm{A}$ is stable.

At point $\mathrm{B}$, the normal force is doubled compared with point A. The contact force is twice as high. and the slope of the contact force characteristic is twice as steep. The critical slope of the mode $S_{c, i}$, however, is constant and now less steep than the contact force characteristic. Again following the logics of equations (5) and (6), the system is unstable leading to self-excitation. A similar argumentation can be applied to point $\mathrm{C}$ that is stable.

2.2. Estimation of Amplitudes in Self-Excited Systems. The typical velocity-dependent contact force or torque characteristic is a superposition of frictional forces (sticking and sliding friction) and viscous forces. Generally, a positive slope is dissipative and a negative is exciting the system. The overall energy consumed or added in one period of the vibrations is given by the time-integral of the multiplication of the velocity-dependent contact force and the velocity and is zero in case of a limit cycle:

$$
\Delta E=\int_{0}^{T} S(\dot{x}) \dot{x} \mathrm{~d} t=0 .
$$

The slope $S(\dot{x})$ is steep and positive for low relative velocity values $\dot{x}$ close to zero (Figure 1 ). The reason is that the contact forces are dependent on the sign of the relative velocity. If the dissipative energy of this part is assumed to be dominant and the amplitude is approximated by a single harmonic $\dot{x}=\widehat{\dot{x}} \sin \left(\omega_{0} t\right)$, an upper amplitude [12] for the limit cycle

$$
\widehat{x}_{i, k}=\frac{v_{0}}{\omega_{0, i}} \cdot \frac{\varphi_{i, k}}{\varphi_{i, j}},
$$

can be defined based on the assumption that the velocity fluctuation equals the mean velocity, $\widehat{\dot{x}}_{i, k}=v_{0}$. The loads and amplitudes are scaled by the ratio of the mode shape amplitudes $\varphi_{i, j} / \varphi_{i, k}$ to a point or node $k$ within the structure. Again one contributing mode $i$ is assumed. This assumption is valid because the dissipation of energy at low relative velocities is not only limiting the energy in the dominant mode $i$ but also in all other modes that are typically completely suppressed. The approach leads to a simple and valid upper limit for the amplitude at any position of an elastic structure as a function of the modal properties of the mode.

2.3. Additional Damping Elements. The influence of different types of linear or nonlinear dampers such as fluid dampers, friction dampers, tuned-mass dampers can be investigated using modal models of the structure. The assumptions for the derivation of the analytical equations are a negligible influence of the forces and the damper device on the mode. Further, the interaction with other modes is negligible and no other modes are considerably excited due to other mechanisms or higher harmonic contents of nonlinear forces.

The model consists of two degrees of freedom (DOF) such as one modal DOF with the modal amplitude $q_{i}$ representing the structure and one physical DOF $x_{\mathrm{d}}$ representing the physical displacement of the damper that is attached at the degree of freedom $j$. The physical displacement of the structure can be calculated by multiplication of the mode shape amplitude $\varphi_{i, j}$ at the position or node $j$ where the damper is forcedly connected to the structure. The 
equation of motion accounting for linear and nonlinear damping forces yields

$$
\left(\begin{array}{ll}
1 & 0 \\
0 & J
\end{array}\right)\left(\ddot{q}_{i} \ddot{x}_{\mathrm{d}}\right)+\left(\begin{array}{cc}
2 D_{i} \omega_{0, i}+\varphi_{i, j}^{2} d & -\varphi_{i, j} d \\
-\varphi_{i, j} d & d
\end{array}\right)\left(\begin{array}{c}
\dot{q}_{i} \\
\dot{x}_{\mathrm{d}}
\end{array}\right)+\left(\begin{array}{cc}
\omega_{0, i}^{2}+\varphi_{i, j}^{2} c & -\varphi_{i, j} c \\
-\varphi_{i, j} c & c
\end{array}\right)\left(\begin{array}{c}
q_{i} \\
x_{\mathrm{d}}
\end{array}\right)=\left(\begin{array}{c}
-\varphi_{i, k} M_{R} \\
M_{R}
\end{array}\right) .
$$

The damper is characterized through a viscous damper constant $d$, a linear stiffness $c$, and an inertia $J$ of the damper. The nonlinear forces $M_{\mathrm{R}}$ of the damper can act on the position or node $k$ of the structure and the inertia mass, respectively. The equation of motion is solved analytically or semianalytically for the different damper types. Herein, the equivalent modal damping ratio $D_{\text {eq }, i}$ for a considered mode $i$ and the contributing critical parameters are derived and analyzed. In the following section, the analytical solutions of different damper principles are discussed. Figure 2 shows the qualitative curve of the damping provided by the various damper principles with respect to frequency (Figure 2, left) and amplitude (Figure 2, right). With respect to the frequency, the robustness of the damping effect is determined, whereas the change in amplitude can be used to make statements about the linearity. Thus, the fluid and tunedmass dampers are linear while the inertia friction and friction bar dampers are nonlinear.

2.3.1. Inertia-Based Friction Damper. A rigid inertia is connected by frictional forces only to the structure in case of an inertia-based damper. Equation (9) is analyzed with the parameters $d=0, c=0$, and $J>0$ and a frictional force $M_{\mathrm{R}}=\widehat{F}_{\mathrm{R}} \operatorname{sign}\left(\varphi_{i, j} \dot{q}_{i}-\dot{x}_{d}\right)$. The derivation of the equivalent damping for the nonlinear system is described in detail the appendix. The general approach is representative for all damper principles discussed. An analytical solution [14] for the dissipated energy and the resulting equivalent modal damping ratio over a period are determined by assuming a harmonic motion of the $i$ th mode $q_{i}=\widehat{q}_{i} \sin \left(\omega_{0, i} t\right)$ and using methods like averaging or harmonic linearization. Due to the nonlinearity, the damping coefficient that can be calculated for the friction damper is dependent on the modal amplitude $\widehat{q}_{i}$ of the considered mode (Figure 2, right). By determining the amplitude dependent optimum $d D_{\mathrm{eq}, i} / d \widehat{q}_{i}=0$, the maximum provided damping and the corresponding amplitude can be determined as follows:

$$
\begin{aligned}
D_{\mathrm{eq}, \max , i} & =\frac{2}{\pi^{2}} \varphi_{i, j}^{2} J, \\
\widehat{q}_{\max , i} & =\frac{\pi \widehat{F}_{\mathrm{R}}}{\sqrt{2} \varphi_{i, j} J \omega_{0, i}^{2}} .
\end{aligned}
$$

The solution shows that the damping is especially dependent on the amplitude of the mode shape $\varphi_{i, j}^{2}$ and linearly dependent on the mass moment of inertia $J$.
2.3.2. Stiffness-Based Friction Damper. The stiffness-based damper [8] bridges the amplitude at two points of the structure through a stiffness $c$. To investigate a stiffnessbased friction damper, equation (9) is used with $d=0, J>0$, and $M_{\mathrm{R}}=\widehat{F}_{\mathrm{R}} \operatorname{sign}\left(\varphi_{i, j} \dot{q}_{i}-\dot{x}_{\mathrm{d}}\right) \quad$ and with $c \gg J \omega_{0, i}^{2}$ and $j \neq k$. In this case, the influence of the stiffness on the dynamic behavior is dominant in comparison to the inertia. The effect of the friction damper is thus dominated by the stiffness and the displacement between the points $j$ and $k$. Again a monoharmonic motion is assumed. In addition, due to the comparably low inertia $J$, the inherent dynamics of the damper can be neglected for the derivation of the analytical solution [14]. The nonlinearity of the friction force leads to an amplitude dependency of the damping coefficient (Figure 2, right). The maximum of the damping ratio and the corresponding amplitude are determined by

$$
\begin{aligned}
\frac{\mathrm{d} D_{\mathrm{eq}, i}}{\mathrm{~d} \widehat{q}_{i}} & =0 \text { gives, } \\
D_{\mathrm{eq}, \max , i} & =\frac{c\left(\varphi_{i, j}-\varphi_{i, k}\right)^{2}}{2 \pi \omega_{0, i}^{2}}, \\
\widehat{q}_{\max i} & =\frac{2 \widehat{F}_{\mathrm{R}}}{c\left|\varphi_{i, j}-\varphi_{i, k}\right|} .
\end{aligned}
$$

The maximum of the damping is increasing with the power of two with the difference of the amplitudes of the mode shape. The stiffness $c$ is contributing linearly to the damping. However, the stiffness may not be excessively increased to limit the effect on the mode shape and the amplitude difference. The stiffness-based damper is dependent on the angular natural frequency of the system and especially effective low frequencies whereas the inertia-based damper provides constant damping for the whole frequency range. The decisive difference is that the contact force of the stiffness-based damper is controlled by the displacement in comparison to the acceleration in case of the inertia-based damper.

2.3.3. Fluid Damper. The parameters of the ordinary fluid damper are $d>0, c=0, J>0$, and $M_{\mathrm{R}}=0$. Assuming harmonic motion of the structure, an analytical solution for the damping 

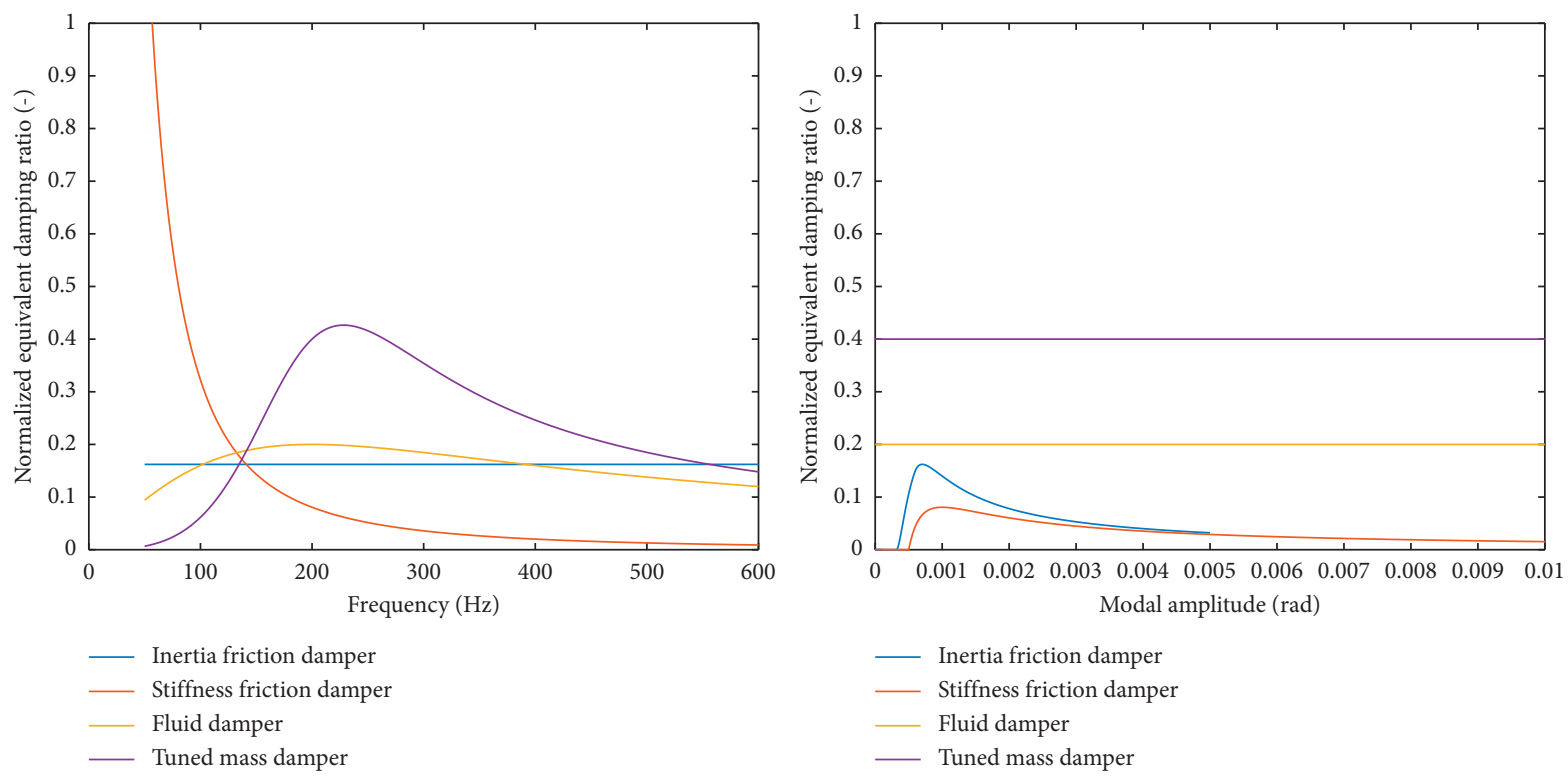

FIGURE 2: Damping as a function of the natural frequency of the underlying structure (a) and as a function of the modal amplitude of the underlying structure at a natural frequency of $200 \mathrm{~Hz}(\mathrm{~b})$.

$$
D_{\mathrm{eq}, i}=\frac{d \omega_{0, i} J^{2} \varphi_{i, j}^{2}}{2\left(d^{2}+\omega^{2} J^{2}\right)},
$$

is obtained. With $\mathrm{d} D_{\text {eq,i }} / \mathrm{d} \omega=0$, an optimal damping ratio regarding an associated frequency

$$
\begin{aligned}
D_{\text {eq, } \max , i} & =\frac{1}{4} \varphi_{i, j}^{2} J, \\
\omega_{0, i} & =\frac{d}{J},
\end{aligned}
$$

is derived. Similar to the friction damping, the equation incorporates one of the most important factors that is the placement with respect to the mode shape represented by the value of $\varphi_{i, j}$ that is contributing to the damping with the power of two. The damping effect prevails over the entire amplitude range of the structural vibration. For a lumped mass model, similar results can be derived that do not contain any information regarding the placement of the damper [6].

2.3.4. Tuned Mass Damper. By adding a stiffness $c>0$ to the fluid damper, the natural frequency of the damper system can be tuned to the vibrational frequency of the underlying structure. The damping of the resulting tuned-mass damper (TMD) is strongly dependent on the frequency (Figure 2, left) [15]. Based on the previously discussed assumptions, the relative movement between the inertia of the damper and the underlying elastic structure is mathematically similar to a system with a mass imbalance. Hence, an analytical approximation of the equivalent modal damping

$$
D_{\mathrm{eq}, i}=\frac{d \varphi_{i, j}^{2}}{2 \omega_{0, i}} \frac{\omega_{0, i}^{4} / \omega_{\mathrm{T}}{ }^{4}}{\left(1-\omega_{0, i}^{2} / \omega_{\mathrm{T}}^{2}\right)^{2}+d^{2} \omega_{0, i}^{2} / J^{2} \omega_{\mathrm{T}}{ }^{4}}=\frac{d \varphi_{i, j}^{2}}{2} \frac{\omega_{0, i}^{3}}{\left(\omega_{\mathrm{T}}^{2}-\omega_{0, i}^{2}\right)^{2}+d^{2} \omega_{0, i}^{2} / J^{2}},
$$

of the modal damping ratio of the $i$ th mode provided by the TMD can be determined. The frequency ratio $\left(\omega_{0, i}\right.$ is the vibration frequency of the structure; $\omega_{\mathrm{T}}=\sqrt{c / m}$ is the natural frequency of the TMD) is particularly important. The damping provided can be approximated for different exceptional cases of the characteristic TMD frequency $\omega_{T}$ to 
TABLE 1: References, comparison of analytical solutions using modal and lumped mass equations, and illustrations of the examined cases.

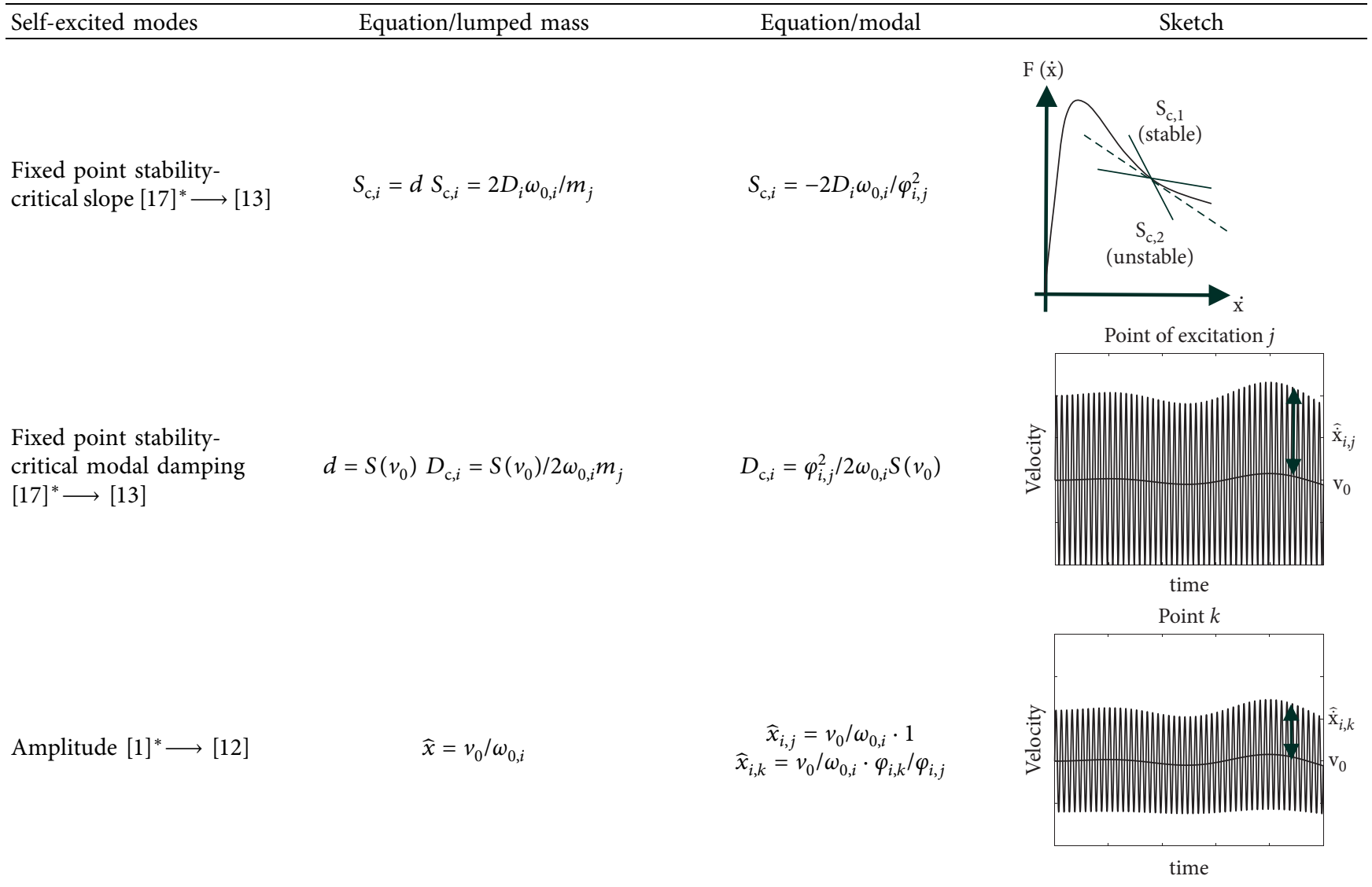

Damping

Friction inertia damper

$[7]^{*} \longrightarrow[14]$

$$
D_{\mathrm{eq}, \max , i}=2 / \pi^{2} m_{j} J
$$

$D_{\mathrm{eq}, \max , i}=2 / \pi^{2} \varphi_{i, j}^{2} J$

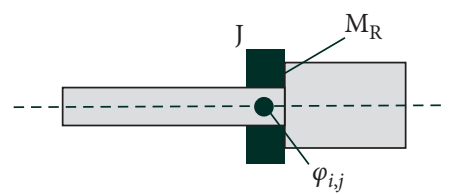

Fluid inertia damper [6]*

$$
D_{\text {eq, } \max , i}=1 / 4 m_{j} J
$$$$
D_{\text {eq, } \max , i}=1 / 4 \varphi_{i, j}^{2} J
$$

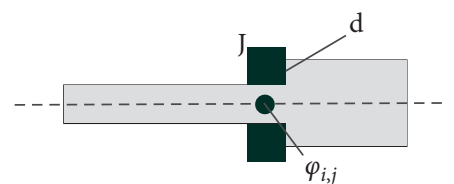

Tuned-mass inertia damper $[9,10]^{*} \longrightarrow[15]^{*}$

$$
\lim _{\omega_{T} \longrightarrow \Omega} D_{\mathrm{eq}, i}=J^{2} \omega_{0, i} / 2 d m_{j}
$$$$
\lim _{\omega_{d} \longrightarrow \Omega} D_{\mathrm{eq}, i}=\varphi_{i, j}^{2} J^{2} \omega_{0, i} / 2 d
$$

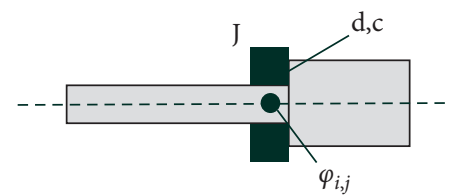

Friction stiffness damper $[8]^{*} \longrightarrow[14]$

$$
D_{\text {eq, } \max , i}=c / 2 \pi \omega_{0, i}^{2} m_{j} \text { (firmly }
$$
clamped on one side)

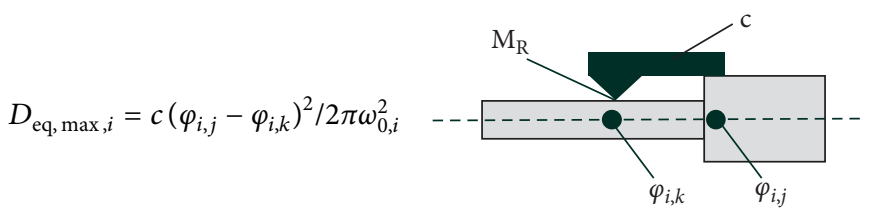




$$
\begin{aligned}
\lim _{\omega_{\mathrm{T}} \longrightarrow 0} D_{\mathrm{eq}, i} & =\frac{d \varphi_{i, j}^{2} \omega_{0, i} J^{2}}{2\left(\omega_{0, i}^{2} J^{2}+d^{2}\right)}, \\
\lim _{\omega_{\mathrm{T}} \longrightarrow \omega_{0, i}} D_{\mathrm{eq}, i} & =\frac{\varphi_{i, j}^{2} J^{2} \omega_{0, i}}{2 d}, \\
\lim _{\omega_{\mathrm{T}} \longrightarrow \infty} D_{\mathrm{eq}, i} & =0 .
\end{aligned}
$$

Equation (15) shows that for small natural frequencies of the TMD $\left(\omega_{\mathrm{T}} \longrightarrow 0\right)$, i.e., for systems where $(c \longrightarrow 0)$, the resulting damping corresponds to the damping of the fluid damper. If the stiffness is comparably high $\left(\omega_{\mathrm{T}} \longrightarrow \infty\right)$, the damping effect of the TMD tends to 0 . The frequency with the highest damping is at $\omega_{\mathrm{T}} \approx \omega_{0, i}$ if the inertia of the damper is rather small [9]. Besides the vibration frequency and the position of the TMD, the inertia and the damping constant are decisive. It should be noted that unrealistically high inertias or too low damping constants significantly influence the system behavior and thus render the approximated solution unsuitable.

\section{Conclusions}

In structural dynamics, several analytical and numerical methods exist to calculate the linear or nonlinear response of the system and to estimate system properties. Analytical equations have limited applicability but they are commonly used to gain direct insight. Single or two degrees of freedom lumped mass models are commonly used for this purpose. In the article, modal models are proposed that are based on exactly the same math.

The article discusses the benefits of a modal description for insights into various problems where the resulting linear or nonlinear equation of motion can be solved analytically or semianalytically. It is shown that the major benefit of the modal description is the visibility of the influence of the modal parameters such as the amplitude of the mode shape at a point of interest. Therefore, the point of observation of an amplitude or the placement of a damper can be derived at every position of a $3 \mathrm{D}$-structure without any changes to the equations that are used. Exemplary results that oppose the results of a lumped mass models and modal models are summarized in Table 1.

\section{Appendix}

The analytical solution for the equivalent damping coefficient of an inertia friction damper [14] with the equation of motion according to equation (9) with $d=0, c=0$, and $J>0$ and a sliding friction force $F_{\mathrm{R}}=\widehat{F}_{\mathrm{R}} \operatorname{sign}\left(\varphi_{i, j} \dot{q}_{i}-\dot{x}_{\mathrm{d}}\right)$ is derived using the harmonic balance method [1]. Herein, a harmonic amplitude of the $i$ th mode $q_{i}=\widehat{q}_{i} \sin \left(\omega_{0, i} t\right)$ is assumed. Based on this assumption, the relative displacement/velocity in the friction contact and subsequently the dissipated energy are estimated. For this purpose, the times $t_{1}, t_{2}, t_{3}$, and $t_{4}$ that correspond to the transitions between sticking and sliding friction in one period of vibration are calculated. For modal amplitudes $\left|\widehat{q}_{i}\right|<\widehat{F}_{\mathrm{R}} / \varphi_{i, j} J \omega_{0, i}^{2}$, the inertia force of the damper $J \omega_{0, i}^{2} \widehat{q}_{i}$ is smaller than the sticking friction force limit $\widehat{F}_{\mathrm{R}}$ and only sticking occurs in one oscillation period with $T=2 \pi / \omega_{0, i}$. Hence, no energy is dissipated $\left(E_{\mathrm{d}}=0\right)$. For higher amplitudes, the dissipated energy in one oscillation period

$E_{\mathrm{d}}=\int_{t_{1}}^{t_{2}} F_{\mathrm{R}} v_{\text {rel }}(t) \mathrm{d} t+\int_{t_{2}}^{t_{3}} 0 \mathrm{~d} t+\int_{t_{3}}^{t_{4}} F_{\mathrm{R}} v_{\text {rel }}(t) \mathrm{d} t+\int_{t_{4}}^{t_{1}+2 \pi / \omega_{0, i}} 0 \mathrm{~d} t$,

can be calculated by four integrals that correspond to sticking and sliding regimes. The energy is only dissipated in the sliding regimes $\left[t_{1} ; t_{2}\right]$ and $\left[t_{3}=t_{1}+\pi / \omega_{0, i}\right.$; $\left.t_{4}=t_{2}+\pi / \omega_{0, i}\right]$. The transition times can be calculated analytically and semianalytically as described in [14]. Generally, a modal dissipated energy $E_{\mathrm{d} \text {,lin }}$ of a linear system in one period and the corresponding linear modal damping ratio $D_{\operatorname{lin}, i}$ for one mode $i$ are determined by

$E_{\mathrm{d}, \text { lin }}=\int_{0}^{2 \pi / \omega_{0, i}} 2 D_{\mathrm{eq}, i} \omega_{0, i} \dot{q}_{i}^{2} \mathrm{~d} t \longrightarrow D_{\mathrm{lin}, i}=\frac{E_{\mathrm{d}, \mathrm{lin}}}{2 \omega_{0, i} \int_{0}^{2 \pi / \omega_{0, i}} \dot{q}_{i}^{2} d t}$.

By assuming the linear dissipated energy $E_{\mathrm{d} \text {,lin }}$ and the nonlinear dissipated energy $E_{\mathrm{d}}$ are equal, the equivalent damping ratio

$$
D_{\mathrm{eq}, i}=\frac{E_{\mathrm{d}}}{2 \pi \omega_{0, i}^{2} \hat{q}_{i}^{2}},
$$

can be estimated. By solving equation (A1) (cf. [14]) and inserting the resulting equation into equation (A3), an analytical solution with

$$
D_{\mathrm{eq}, i}=\frac{2 \widehat{F}_{\mathrm{R}} \phi_{i, j}}{\pi \omega_{0, i}^{2} q \widehat{q}_{i}} \sqrt{1-\left(\frac{\pi \widehat{F}_{\mathrm{R}}}{2 J \widehat{q}_{i} \phi_{i, j} \omega_{0, i}^{2}}\right)^{2}},
$$

is derived with an amplitude dependent maximum at $D_{\text {eq, } \max , i}=2 / \pi^{2} \phi_{i, j}^{2} J$ at $\widehat{q}_{\max , i}=\pi \widehat{F}_{\mathrm{R}} / \sqrt{2} \phi_{i, j} J \omega_{0, i}^{2}$.

\section{Data Availability}

The data used to support the findings of this study are included within the article.

\section{Conflicts of Interest}

The authors declare that there are no conflicts of interest regarding the publication of this paper.

\section{Acknowledgments}

The authors would like to thank Baker Hughes for supporting the work and giving permission to publish this paper. The authors acknowledge support by the Open Access Publication Funds of the TU Braunschweig. 


\section{References}

[1] K. Magnus, K. Popp, and W. Sextro, Schwingungen: Physikalische Grundlagen und mathematische Behandlung von Schwingungen, Springer Fachmedien Wiesbaden, Wiesbaden, Germany, 2013.

[2] T. M. Cameron and J. H. Griffin, "An alternating frequency/ time domain method for calculating the steady-state response of nonlinear dynamic systems," Journal of Applied Mechanics, vol. 56, no. 1, pp. 149-154, 1989.

[3] J. A. Sanders, J. Murdock, and F. Verhulst, Averaging Methods in Nonlinear Dynamical Systems, Springer, New York, NY, USA, 2007.

[4] R. R. Craig and M. C. C. Bampton, "Coupling of substructures for dynamic analyses," AIAA Journal, vol. 6, no. 7, pp. 1313-1319, 1968.

[5] M. P. Castanier, Y.-C. Tan, and C. Pierre, "Characteristic constraint modes for component mode Synthesis," AIAA Journal, vol. 39, no. 6, pp. 1182-1187, 2001.

[6] K. E. Hafner and H. Maass, Torsionsschwingungen in der Verbrennungskraftmaschine, Springer, Vienia, Australia, 1985.

[7] I. López, J. M. Busturia, and H. Nijmeijer, "Energy dissipation of a friction damper," Journal of Sound and Vibration, vol. 278, no. 3, pp. 539-561, 2004.

[8] K. Popp, L. Panning, and W. Sextro, "Vibration damping by friction forces: theory and applications," Journal of Vibration and Control, vol. 9, no. 3-4, pp. 419-448, 2003.

[9] J. P. Den Hartog and G. Mesmer, Mechanische Schwingungen, Springer, Berlin, Germany, 1952.

[10] S. V. Bakre and R. S. Jangid, "Optimum parameters of tuned mass damper for damped main system," Structural Control and Health Monitoring, vol. 14, no. 3, pp. 448-470, 2007.

[11] D. J. Ewins, Modal Testing: Theory, Practice and Application, Research Studies Press, Baldock, England, 2000.

[12] A. Hohl, M. Tergeist, H. Oueslati et al., "Prediction and mitigation of torsional vibrations in drilling systems," in Proceedings of the IADC/SPE Drilling Conference and Exhibition, Texas, TX, USA, September 2016.

[13] A. Hohl, M. Tergeist, H. Oueslati et al., "Derivation and experimental validation of an analytical criterion for the identification of self-excited modes in drilling systems," Journal of Sound and Vibration, vol. 342, no. 1, pp. 290-302, 2015.

[14] V. Kulke, G.-P. Ostermeyer, M. Tergeist, and A. Hohl, "Semianalytical approach for derivation of an equivalent modal friction-damping ratio and its application in a self-excited drilling system," Dynamics, vol. 4, 2019.

[15] V. Kulke, P. Thunich, F. Schiefer, and G.-P. Ostermeyer, "A method for the design and optimization of nonlinear tuned damping concepts to mitigate self-excited drill string vibrations using multiple scales lindstedt-poincaré," Applied Sciences, vol. 11, no. 4, p. 1559, 2021.

[16] M. Neubauer and J. Wallaschek, "Vibration damping with shunted piezoceramics: fundamentals and technical applications," Mechanical Systems and Signal Processing, vol. 36, no. 1, pp. 36-52, 2013.

[17] L. P. Shilnikov, A. L. Shilnikov, D. V. Turaev, and L. O. Chua, Methods of Qualitative Theory in Nonlinear Dynamics: Part I, World Scientific Pub. Co, Singapore, 1998. 